\title{
REPRESENTATION OF VECTOR VALUED NONLINEAR FUNCTIONS
}

\author{
BY \\ VICTOR J. MIZEL AND K. SUNDARESAN
}

\begin{abstract}
A representation theorem for "additive" nonlinear functionals on spaces $L^{p}(\mu)$ is here extended to "additive" nonlinear functions from LebesgueBochner function spaces $L_{E}^{p}(\mu)$ ( $E$ a separable Banach space) into Banach spaces $B$. A counterexample is provided to show that the restriction to separable $E$ is essential.
\end{abstract}

Let $(X, \Sigma, \mu)$ be a measure space, $B$ be an arbitrary Banach space, and $E$ be a separable Banach space. Let $M_{E}$ be the vector space of $E$-valued Bochner measurable functions on $X$. A function $F: M_{E} \rightarrow B$ is said to be additive if $F(x+y)$ $=F(x)+F(y)$ whenever $x, y \in M_{E}$ with (almost everywhere) disjoint supports. More specifically we are concerned with continuous additive functions defined on the associated $L_{E}^{p}$ spaces $(1 \leqq p \leqq \infty)$ of Bochner $\mu$-integrable functions. The main problem that arises here is to characterize the additive functions on $L_{E}^{p}(\mu) \rightarrow B$ which admit an integral representation of the form $F(x)=\int_{X} \varphi(x(\xi), \xi) d \mu(\xi)$ where $\varphi$ is required to satisfy certain conditions related to those occurring in the theory of nonlinear integral equations, Krasnosel'skir [5]. Several authors considered this problem when both $E$ and $B$ are the real line, $R$. See for these and related results Mizel [6], Mizel and Sundaresan [7], Drewnowski and Orlicz [2] and Woyczyński [11]. It will be seen that the case $E=R, p=\infty$ is the crucial one. Once the results are available for this case then, by straightforward techniques, one can handle the general case of $F: L_{E}^{p}(\mu) \rightarrow B(1 \leqq p \leqq \infty)$.

The present study is motivated by the following considerations:

1. The representation theorems in [6] and [2] are false in the $B$-valued case even for $E=R$. We furnish an example in $\$ 3$ justifying this assertion.

2. The results in this paper are applicable to investigations based on Lebesgue (pseudo) differentiability of vector valued additive set functions as well as to investigations based on Radon-Nikodym differentiability of such functions. They also provide a sufficient condition that certain types of vector valued random processes have continuous sample paths.

3. Integral representations of the type considered here have, for the case of functionals (i.e. $B=R$ ), already been studied extensively when the functionals are

Received by the editors July 30, 1969 and, in revised form, March 29, 1970.

AMS 1969 subject classifications. Primary 2816, 2850, 4780, 6040.

Key words and phrases. Additive functions, Carathéodory functions, locally uniformly continuous in variation, Lebesgue-Bochner function spaces, Bochner measurable.

Copyright (C) 1971, American Mathematical Society 
linear (Edwards [4, Chapter 8]), and our methods provide an alternate proof for certain of the latter representation theorems (see $\$ 4$ ).

The techniques that are adopted in the proofs of various assertions are very similar to those in [6], [7], and [2], apart from the use of the general RadonNikodym theorem established in a recent paper of Rieffel [10], and of a theorem of Phillips on Lebesgue differentiation.

The paper is divided into four sections. In $\S 1$ after recalling the notations and definitions we proceed to establish the representation theorem for $B$-valued additive functions on $L_{R}^{\infty}(\mu)$ and to deduce from this theorem the representation theorems for the case of $L_{R}^{p}(\mu), 1 \leqq p<\infty$. In $\S 2$ we introduce the Carathéodory differential of a 1-parameter family of additive set functions and obtain a necessary and sufficient condition for the differentiability of such a family in this sense. In $\$ 3$ we provide an example illustrating that the theorems in [6] and [2] are not true in the $B$-valued case even when $B$ is a separable Hilbert space and $E=R$. In $\S 4$ we point out how the results of $\S 1$ can be extended to the case of an arbitrary separable Banach space $E$.

1. Representation theorems. In the sequel $(X, \Sigma, \mu)$ is a complete $\sigma$-finite positive measure space unless otherwise specified and $B$ is an arbitrary Banach space. $R$ is the real line. $L^{\infty}(\mu)$ is the space of equivalence classes of essentially bounded (real-valued) measurable functions $x$, with the norm $\|x\|$ $=$ ess $\sup \{|x(\xi)| \mid \xi \in X\} . \mathscr{L}_{B}^{p}$ is the collection of Bochner $p$-summable $B$-valued functions on $X$ and $L_{B}^{p}(1 \leqq p<\infty)$ is the corresponding Banach space of equivalence classes of $p$-summable functions.

A function $\varphi: R \times X \rightarrow B$ is said to be a Carathéodory function on $X$ to $B$, in short $\varphi \in \operatorname{Car}(X ; B)(\operatorname{Car} B$ when the measure space is understood), if $\varphi(c, \cdot)$ is a Bochner measurable function for each real number $c$ and $\varphi(\cdot, \xi)$ is a continuous function for all $\xi$ outside a $\mu$-null set. A function $F: L^{\infty}(\mu) \rightarrow B$ is called additive ("orthogonally additive" according to [2]) if $F(x+y)=F(x)+F(y)$ for $x, y \in L^{\infty}(\mu)$ with disjoint support, i.e. $x y=0 \mu$.a.e. Given a finitely additive set function $G: \Sigma \rightarrow B$ having finite variation, the variation of $G$ on a set $E \in \Sigma$ is denoted by $V(G)(E)$. If $\left\{G_{t}\right\}_{t \in R}$ is a one parameter family of finitely additive set functions on $\Sigma$ and $d, \delta$ are two positive numbers then $V_{d}(\delta, G)$ denotes the quantity

$$
V_{d}(\delta, G)=\sup \sum_{i=1}^{n} V\left(G_{h_{1}^{1}}-G_{h_{2}^{i}}\right)\left(E_{i}\right)
$$

where the supremum is taken over all ordered $n$-tuples, $n \geqq 1$, of pairwise disjoint measurable sets $\left\{E_{1}, \ldots, E_{n}\right\}$ with $h_{1}^{i}, h_{2}^{i} \in[-d, d]$ and $\left|h_{1}^{i}-h_{2}^{i}\right| \leqq \delta, 1 \leqq i \leqq n$. The family $\left\{G_{t}\right\}$ is said to be locally uniformly continuous in variation provided $\lim _{\delta \rightarrow 0+} V_{d}(\delta, G)=0$ for every $d>0$. Finally, if $F$ is an additive function on $L^{\infty}(\mu)$ and $h$ is a real number then $F_{h}$ will denote the finitely additive set function on $\Sigma \rightarrow B$ defined by $F_{h}(E)=F\left(h I_{E}\right)$ where $I_{E}$ is the characteristic function of $E$. 
We recall certain concepts concerning vector valued set functions studied in detail in [10]. The average range of an additive set function $G: \Sigma \rightarrow B$ on a measurable set $E, 0<\mu(E)<\infty$, is the set

$$
A_{E}(G)=\left\{G\left(E^{\prime}\right) / \mu\left(E^{\prime}\right) \mid \dot{E}^{\prime} \in \Sigma, E^{\prime} \subset E, 0<\mu\left(E^{\prime}\right)\right\} .
$$

A set function $G$ has locally almost compact average range if whenever $E \in \Sigma$, $0<\mu(E)<\infty$, and $\varepsilon>0$ then there exists a measurable set $E_{0} \subset E$ such that $\mu\left(E-E_{0}\right)<\varepsilon$ and $A_{E_{0}}(G)$ is a precompact set in $B$. (See Proposition 1.12 on p. 472 in [10].)

Before proceeding to the representation of the additive functions we state several useful lemmas.

LEMMA 1. If $F: L^{\infty}(\mu) \rightarrow B$ is an additive function such that $\left\{F_{t}\right\}_{t \in R}$ is locally uniformly continuous in variation then $F$ is uniformly continuous on bounded subsets of simple functions.

Proof. Let $S$ be the subspace of $L^{\infty}(\mu)$ consisting of simple functions. Let $d>0$ be a fixed positive number and $S_{d}=\left\{x \mid x \in S,\|x\|_{\infty} \leqq d\right\}$. The hypothesis on $F$ in the lemma implies that for each $\varepsilon>0$ there exists a positive number $\delta(\varepsilon)$ such that if $\delta \leqq \delta(\varepsilon)$ then $V_{d}(\delta, F) \leqq \varepsilon$. Let now $x, y \in S_{d}$ be such that $\|x-y\|_{\infty} \leqq \delta(\varepsilon)$. Thus there exists a finite collection of pairwise disjoint measurable sets $\left\{E_{i}\right\}_{1 \leqq i \leqq n}$ such that $x=\sum a_{i} I_{E_{i}}, y=\sum b_{i} I_{E_{i}}$ with $\left|a_{i}-b_{i}\right| \leqq \delta(\varepsilon)$. Thus

$$
\|F(x)-F(y)\| \leqq \sum_{i=1}^{n}\left\|\left(F_{a_{i}}-F_{b_{i}}\right)\left(E_{i}\right)\right\| \leqq \sum_{i=1}^{n} V\left(F_{a_{i}}-F_{b_{i}}\right)\left(E_{i}\right)<\varepsilon .
$$

REMARK 1. (a) The converse of the assertion in the preceding lemma is false, i.e. the uniform continuity of $F$ on bounded subsets of $S$ need not imply that $\left\{F_{t}\right\}_{t \in R}$ is locally uniformly continuous in variation. This is discussed at the end of the example in $\$ 3$.

(b) For convenience of reference we note that if $f: X \rightarrow B$ is a function in $L_{B}^{1}$ and if, for each $E \in \Sigma, G(E)=\int_{E} f d \mu$ then $V(G)(E)=\int_{E}\|f(\xi)\| d \mu(\xi)$. For a proof of this fact we refer to Dunford and Schwartz [3].

Lemma 2. If $\varphi: R \times X \rightarrow B$ is a Carathéodory function on $X$ to $B$ and $T \in \Sigma$ has finite measure then corresponding to any three preassigned positive numbers $\delta, \varepsilon$ and $A$ there exists a measurable set $M$, and a positive number $\eta$ such that $(1) \mu(M) \leqq \delta$ and (2) for all $\xi \in T \sim M,\left\|\varphi(t, \xi)-\varphi\left(t^{\prime}, \xi\right)\right\| \leqq \varepsilon$ if $\left|t-t^{\prime}\right| \leqq \eta,|t|,\left|t^{\prime}\right| \leqq A$.

Proof. Let us define a sequence of measurable sets $\left\{X_{n}\right\}_{n \geqq 1}$ as follows:

$$
X_{n}=\left\{\xi\left|t, t^{\prime} \in[-A, A],\right| t-t^{\prime} \mid \leqq 1 / n \Rightarrow\left\|\varphi(t, \xi)-\varphi\left(t^{\prime}, \xi\right)\right\| \leqq \varepsilon\right\} .
$$

If $\left\{\left(a_{n}^{i}, b_{n}^{i}\right)\right\}_{i \geqq 1}$ is an enumeration of all rational number pairs in $[-A, A]$ such that $\left|a_{n}^{i}-b_{n}^{i}\right| \leqq 1 / n$ then

$$
X_{n}=\bigcap_{i \geqq 1}\left\{\xi \mid\left\|\varphi\left(a_{n}^{i}, \xi\right)-\varphi\left(b_{n}^{i}, \xi\right)\right\| \leqq \varepsilon\right\}-N_{n},
$$


where $N_{n}$ is a subset of the null set in $X$ for which $\varphi(\cdot, \xi)$ is not continuous. Thus $X_{n}$ is a measurable set for all $n \geqq 1$. Since $\varphi(\cdot, \xi)$ is uniformly continuous on $[-A, A]$ for almost all $\xi$ it follows that $\mu\left(T \sim \bigcup_{n \geqq 1} X_{n}\right)=0$. Since $X_{n} \uparrow T$ there exists an integer $N$ such that $\mu\left(T \sim X_{N}\right) \leqq \delta$ and, for all $\xi \in X_{N},\left\|\varphi(t, \xi)-\varphi\left(t^{\prime}, \xi\right)\right\| \leqq \varepsilon$ if $t, t^{\prime} \in[-A, A]$ and $\left|t-t^{\prime}\right| \leqq 1 / N$. Choosing for $M$ and $\eta, X_{N}$ and $1 / N$ respectively, the proof is completed.

REMARK 2. We note that the dependence of $M$ on the given $\varepsilon$ is inessential. Actually the set $M$ could be chosen independent of $\varepsilon$ and $A$, by utilizing sequences $\left\{A_{n}\right\},\left\{\varepsilon_{n}\right\},\left\{\delta_{n}\right\}$ with $A_{n} \rightarrow \infty, \varepsilon_{n} \rightarrow 0$, and $\sum \delta_{n}<\delta$.

This is easily seen from the proof in the next lemma.

LemMA 3. Let $f: R \rightarrow \mathscr{L}_{B}^{1}$ where $\mathscr{L}_{B}^{1}$ is the space of all B-valued Bochner integrable functions on $X$ and for each $t \in R$ let $F_{t}$ denote the indefinite integral of $f(t)$. Then if the family $\left\{F_{t}\right\}_{t \in R}$ is locally uniformly continuous in variation there exists a function $\varphi \in$ Car $B$ such that, for all $t, f(t)(\xi)=\varphi(t, \xi)$ almost everywhere.

Proof. As a first step we verify that, for each positive number $d, f_{t}(\xi)=f(t, \xi)$ is uniformly continuous on the set of rationals $Q_{d} \subset[-d, d]$ at all $\xi$ outside a $\mu$-null set. Let $\varepsilon>0$ and let

$$
A_{n}=\left\{\xi \mid \text { there exist } t_{1}, t_{2} \in Q_{d} \text { with }\left|t_{1}-t_{2}\right| \leqq 1 / n \text { and }\left\|f\left(t_{1}, \xi\right)-f\left(t_{2}, \xi\right)\right\| \geqq \varepsilon\right\} \text {. }
$$

It is verified that $\left\{A_{n}\right\}$ is a nested family of measurable sets. Further $\lim \mu\left(A_{n}\right)=0$ as $n \rightarrow \infty$. For if $\lim \mu\left(A_{n}\right) \neq 0$ there exists a positive number $a$ such that $\mu\left(A_{n}\right) \geqq a$. Given $\delta>0$ let $n$ be chosen so that $1 / n \leqq \delta$. Let $\left\{\left(p^{i}, q^{i}\right)\right\}_{i \geqq 1}$ be an enumeration of all rational pairs in $Q_{d} \times Q_{d}$ such that $\left|p^{i}-q^{i}\right| \leqq 1 / n$ and let

$$
A_{n}^{i}=\left\{\xi \mid\left\|f\left(p^{i}, \xi\right)-f\left(q^{i}, \xi\right)\right\| \geqq \varepsilon\right\} .
$$

Let $C_{n}^{i+1}=A_{n}^{i+1} \sim \bigcup_{j \leqq i} A_{n}^{j}$. Thus $a \leqq \mu\left(A_{n}\right)=\sum_{i=1}^{\infty} \mu\left(C_{n}^{i}\right)$. Let $N$ be so chosen that $\sum_{i=1}^{N} \mu\left(C_{n}^{i}\right) \geqq a / 2$. Now $\sum_{i=1}^{N} \int_{C_{n}^{i}}\left\|f\left(p^{i}, \xi\right)-f\left(q^{i}, \xi\right)\right\| d \mu(\xi) \geqq a \varepsilon / 2$. Hence by Remark 1(b) it follows that $\lim _{\delta \rightarrow 0+} V_{d}(\delta, F) \neq 0$. That is the family $\left\{F_{t}\right\}_{t \in R}$ is not locally uniformly continuous in variation contrary to the hypothesis. Thus if $A^{\varepsilon}=\bigcap_{n \geqq 1} A_{n}$ then $\mu\left(A^{\varepsilon}\right)=0$. Now let $B_{d}=\bigcup_{m \geqq 1} A^{1 / m}$. Then $\mu\left(B_{d}\right)=0$ and if $\xi \notin B_{d}, f(\cdot, \xi)$ is uniformly continuous on the set $Q_{d}$. Let us extend the function $f(\cdot, \xi)$ at each $\xi \notin B_{d}$ to a function $\varphi_{d}(\cdot, \xi)$ where $\varphi_{d}(\cdot, \xi)$ is a continuous function on the interval $[-d, d]$. Such a function is uniquely determined by $f(\cdot, \xi)$ since $Q_{d}$ is dense in $[-d, d]$. Let now $\varphi(\cdot, \xi)=\bigcup_{n \geqq 1} \varphi_{n}(\cdot, \xi)$ for $\xi \in X \sim \bigcup_{n \geqq 1} B_{n}$, and $\varphi(\cdot, \xi)=0$ for $\xi \in \bigcup_{n \geqq 1} B_{n}$. Since $\mu\left(\bigcup_{n \geqq 1} B_{n}\right)=0$ it follows that for all rational numbers $t$ and for $\xi \notin \bigcup_{n \geqq 1} B_{n}, f(t)(\xi)=\varphi(t, \xi)$.

From this it follows that $F_{t}(E)=\int_{E} \varphi(t, \xi) d \mu(\xi)$ for all $E \in \Sigma$ and $t \in R$. For let $t \in R$ and let $t_{n}$ be a sequence of rationals such that $t_{n} \rightarrow t$. From the local uniform continuity in variation of $\left\{F_{t}\right\}_{t \in R}$ it follows that (see Remark 1(b))

$$
\int_{E}\left\|f\left(t_{n}, \xi\right)-f(t, \xi)\right\| d \mu(\xi) \rightarrow 0 .
$$


Thus the functions $f\left(t_{n}, \cdot\right)$, and therefore the functions $\varphi\left(t_{n}, \cdot\right)$ are uniformly bounded in $L_{B}^{1}$ norm on $E$ and in addition have the property that their indefinite integrals are uniformly absolutely continuous (Theorem 6, p. 122 in [3]), i.e. $\int_{U}\left\|f\left(t_{n}\right)(\xi)\right\| d \mu(\xi) \rightarrow 0$ for $U \subset E$ as $\mu(U) \rightarrow 0$, uniformly in $n$. In addition, the functions $\varphi\left(t_{n}, \cdot\right)$ satisfy $\varphi\left(t_{n}, \xi\right) \rightarrow \varphi(t, \xi)$ a.e. Thus by Vitalli's convergence theorem $[3$, p. 150$]$, equicontinuity being clear,

$$
\begin{aligned}
F_{t}(E) & =\int_{E} f(t, \xi) d \mu(\xi)=\lim \int_{E} f\left(t_{n}, \xi\right) d \mu(\xi) \\
& =\lim \int_{E} \varphi\left(t_{n}, \xi\right) d \mu(\xi)=\int_{E} \varphi(t, \xi) d \mu(\xi) .
\end{aligned}
$$

Since this holds for all $E \in \Sigma, \varphi(t, \xi)=f(t, \xi)$ a.e. which completes the proof.

REMARK 3. Lemma 3 has an obvious interpretation in probability theory. It provides a sufficient condition that a $B$-valued random function ("stochastic process") $f$ on the real axis, with values $f(t)$ in $\mathscr{L}_{B}^{1}(X)$, be equivalent to ("have a version as") a $B$-valued random function $\varphi$ possessing almost surely continuous sample paths. On the other hand this condition is not necessary, as is seen by the following example with $B=R$ and with $X=$ the interval $0 \leqq \xi \leqq 1$ with Lebesgue measure. We take

$$
\begin{aligned}
f(t, \xi)=\varphi(t, \xi) & =t^{-1}, & & |\xi-2 t|<t, \\
& =|\xi-2 t|^{-1} k(t, \xi), & & |\xi-2 t| \geqq t,
\end{aligned}
$$

where $k$ is a smooth function on $R \times[0,1]-\{(0,0)\}$ which is identically 1 for $|\xi-2 t| \leqq 3 / 2 t$ and identically 0 for $|\xi-2 t| \geqq 2 t$. Then with $h_{1}^{1}=\delta / 3, h_{2}^{1}=0$ and $E_{1}=(2 \delta / 3, \delta)$ one deduces that $V_{d}(\delta, F) \geqq 1$ for all $\delta$. (Conditions of a different type are discussed in [15, Chapter III].)

We next proceed to the key representation theorem of the paper. We state as separate theorems the necessary and sufficient conditions guaranteeing the representation.

THEOREM 1. Let $F$ be an additive function on $L^{\infty}(\mu)$ into a Banach space $B$ such that

(1) $x_{n} \rightarrow x$ boundedly a.e. $\Rightarrow\left\|F\left(x_{n}\right)-F(x)\right\| \rightarrow 0$,

(2) for each real number $t, F_{t}$ has almost locally compact average range,

(3) for each real number $t, V\left(F_{t}\right)$ is a finite measure,

(4) the family $\left\{F_{t}\right\}_{t \in R}$ is locally uniformly continuous in variation.

Then there exists a function $\varphi \in \operatorname{Car} B$ such that for all $x \in L^{\infty}(\mu)$ the function $\varphi \circ x(\xi)=\varphi(x(\xi), \xi)$ is in $L_{B}^{1}$, and

$$
F(x)=\int_{X} \varphi(x(\xi), \xi) d \mu(\xi) .
$$

Further such a representing function $\varphi$ can be chosen to satisfy $\varphi(0, \xi)=0$ for $\xi$ a.e. and is then unique up to sets of the form $R \times N$ where $N$ is a $\mu$-null set. 
Proof. Condition (1) implies that for each real number $t$ the set function $F_{t}$ is countably additive. Thus $F_{t}$ is a $B$-valued measure. By the "main theorem" and the remarks on p. 479 in [10] our conditions (2) and (3) imply that for every real number $t$ there exists a function $f_{t} \in \mathscr{L}_{B}^{1}$ uniquely defined up to null sets such that $F_{t}(E)=\int_{E} f_{t}(\xi) d \mu(\xi)$ for all $E \in \Sigma$. Since the family of set functions $\left\{F_{t}\right\}_{t \in R}$ satisfy the conditions in the preceding lemma it follows that there exists a function $\varphi \in \operatorname{Car} B$ such that for all real numbers $t$ and for all sets $E \in \Sigma$

$$
F_{t}(E)=F\left(t I_{E}\right)=\int_{E} \varphi(t, \xi) d \mu(\xi)
$$

Thus by additivity of $F$, if $x$ is a simple function, $F(x)=\int_{X} \varphi(x(\xi), \xi) d \mu(\xi)$. Let now $x \in L^{\infty}(\mu)$. Then there exists a sequence $\left\{x_{n}\right\}_{n \geqq 1}$ of simple functions in $L^{\infty}(\mu)$ such that $\left\|x_{n}-x\right\|_{\infty} \rightarrow 0$ and $x_{n}(\xi) \rightarrow x(\xi)$ a.e. Since $\varphi \in \operatorname{Car} B$ it is verified that $\varphi\left(x_{n}(\xi), \xi\right) \rightarrow \varphi(x(\xi), \xi)$ a.e. Further, condition (4) on $F$ together with the fact that $\left\{x_{n}\right\}_{n \geqq 1}$ is a Cauchy sequence in $L^{\infty}(\mu)$ implies that $\left\{\varphi\left(x_{n}(\xi), \xi\right)\right\}_{n \geqq 1}$ is a Cauchy sequence in $L_{B}^{1}$. Hence it follows that $\int_{X} \varphi\left(x_{n}(\xi), \xi\right) d \mu(\xi) \rightarrow \int_{X} \varphi(x(\xi), \xi) d \mu(\xi)$. Thus by condition (1) $F(x)=\int_{X} \varphi(x(\xi), \xi) d \mu(\xi)$. Note that by the construction $\varphi(0, \xi)=0$ a.e.

For the proof of the uniqueness assertion in the theorem observe that for any $\bar{\varphi} \in \operatorname{Car} B$ giving a representation of $F$ similar to that provided by the above function $\varphi$, one has

$$
\begin{aligned}
F_{t}(E) & =F\left(t I_{E}\right)=\int_{X} \bar{\varphi}\left(t I_{E}(\xi), \xi\right) d \mu(\xi) \\
& =\int_{E}(\bar{\varphi}(t, \xi)-\bar{\varphi}(0, \xi)) d \mu(\xi)+\int_{X} \bar{\varphi}(0, \xi) d \mu(\xi) .
\end{aligned}
$$

Thus, since $F(0)=0$ it is necessary that (1) $\int_{X} \bar{\varphi}(0, \xi) d \mu(\xi)=0$, and (2) for each $t \in R, \bar{\varphi}(t, \cdot)-\bar{\varphi}(0, \cdot)$ is a Radon-Nikodym derivative of $F_{t}$. The requirement that $\bar{\varphi}(0, \cdot)=0$ a.e. thus implies that $\bar{\varphi}(t, \xi)=\varphi(t, \xi)$ for each rational $t$ and each $\xi \notin N_{0}$ where $N_{0}$ is a null set. It then follows by definition of Car $B$ that $\bar{\varphi}=\varphi$ except on a set $R \times N$, as asserted.

We proceed next to establish the converse of the preceding theorem.

Theorem 2. Let $\varphi \in \operatorname{Car} B$ be such that (1) $\varphi(0, \xi)=0$ for $\xi$ a.e. and (2) the function $\varphi \circ x(\xi)=\varphi(x(\xi), \xi)$ is in $L_{B}^{1}$ for all $x \in L^{\infty}(\mu)$. If for $x \in L^{\infty}(\mu)$ one defines

$$
F(x)=\int_{X} \varphi(x(\xi), \xi) d \mu(\xi)
$$

then $F$ is an additive function satisfying the conditions (1) to (4) of the preceding theorem.

Proof. Let $\left\{x_{n}\right\}_{n \geqq 1}$ be a sequence of functions in $L^{\infty}(\mu)$ such that $x_{n}(\xi) \rightarrow x(\xi)$ boundedly a.e. Note that if $\psi(t, \xi)=\|\varphi(t, \xi)\|$ then $\psi \in \operatorname{Car} R$ and the function 
$x \rightarrow \psi \circ x$ maps $L^{\infty}(\mu) \rightarrow L^{1}(\mu)$. Thus by the proof of Theorem 1 in Mizel [6] it follows that

$$
\|\varphi\| \circ x_{n}=\psi \circ x_{n} \rightarrow \psi \circ x=\|\varphi\| \circ x \text { in } L^{1}(\mu) \text { norm. }
$$

Hence the indefinite integrals $\int \varphi\left(x_{n}(\xi), \xi\right) d \mu(\xi)$ are uniformly absolutely continuous. Moreover $\varphi \circ x_{n} \rightarrow \varphi \circ x$ a.e. Thus by Vitalli's theorem (DunfordSchwartz [3]) $\left\|F\left(x_{n}\right)-F(x)\right\| \rightarrow 0$. By the main theorem in [10] it follows that $F$ satisfies conditions (2) and (3). We proceed to verify (4). Using Remark 1(b) it can be seen that it is enough to verify that the mapping $x \rightarrow \varphi \circ x$ of $L^{\infty}(\mu)$ into $L_{B}^{1}$ is uniformly continuous on bounded subsets of simple functions in $L^{\infty}(\mu)$. Assume now the following: (A) there exist positive numbers $d$ and $a$ and two sequences of simple functions $\left\{x_{n}\right\},\left\{y_{n}\right\}$ such that $\left\|x_{n}-y_{n}\right\|_{\infty} \leqq 1 / n,\left\|x_{n}\right\|_{\infty},\left\|y_{n}\right\|_{\infty} \leqq d$, but $\int_{X}\left\|\varphi \circ x_{n}-\varphi \circ y_{n}\right\| d \mu \geqq a$ for all $n$. Let us consider first the case in which $(X, \Sigma, \mu)$ is a finite measure space.

Let $\delta_{1}>0$ be so chosen that if $E \in \Sigma, \mu(E)<2 \delta_{1}$, then $\int_{E}\left\|\varphi \circ x_{1}-\varphi \circ y_{1}\right\| d \mu \leqq a / 3$. Such a $\delta_{1}$ certainly exists since the indefinite integral $\int\left\|\varphi \circ x_{1}-\varphi \circ y_{1}\right\| d \mu$ is $\mu$-absolutely continuous. Corresponding to the pair of real numbers $\left(\delta_{1}, a / 3 \mu(X)\right)$ it follows from Lemma 2 that there exists a measurable set $S_{1}$ and an integer $n_{2}$ such that $\mu\left(X-S_{1}\right) \leqq \delta_{1}$ and for $\xi \in S_{1},\left\|\varphi(t, \xi)-\varphi\left(t^{\prime}, \xi\right)\right\| \leqq a / 3 \mu(X)$ if $\left|t-t^{\prime}\right| \leqq 1 / n_{2}$, $|t|,\left|t^{\prime}\right| \leqq d$. Hence by the choice of $\left\{x_{n}\right\},\left\{y_{n}\right\}$ it follows that $\int_{X-s_{1}}\left\|\varphi \circ x_{n_{2}}-\varphi \circ y_{n_{2}}\right\| d \mu$ $\geqq 2 a / 3$ and $\int_{X-S_{1}}\left\|\varphi \circ x_{1}-\varphi \circ y_{1}\right\| d \mu \leqq a / 3$. Thus if $S_{2}=X-S_{1}$ then $S_{1}, S_{2}, n_{1}=1$ and $n_{2}$ satisfy

$$
\begin{array}{ll}
\int_{S_{1}}\left\|\varphi \circ x_{n_{1}}-\varphi \circ y_{n_{1}}\right\| \geqq \frac{2}{3} a, & \int_{S_{2}}\left\|\varphi \circ x_{n_{1}}-\varphi \circ y_{n_{1}}\right\| \leqq \frac{1}{3} a, \\
\int_{S_{2}}\left\|\varphi \circ x_{n_{2}}-\varphi \circ y_{n_{2}}\right\| \geqq \frac{2}{3} a, & \mu\left(S_{2}\right) \leqq \delta_{1} \leqq \frac{1}{2} \mu\left(S_{1}\right) .
\end{array}
$$

Suppose we have selected functions $\left\{x_{n_{i}}\right\},\left\{y_{n_{i}}\right\}$ and measurable sets $S_{i}, 1 \leqq i \leqq k$, as well as positive numbers $\delta_{i}, 1 \leqq i \leqq k-1$, such that

(a) $\mu\left(S_{i}\right) \leqq \delta_{i-1} \leqq \frac{1}{2} \mu\left(S_{i-1}\right)$,

(b) $E \in \Sigma$ and $\mu(E) \leqq 2 \delta_{i} \Rightarrow \int_{E}\left\|\varphi \circ x_{n_{i}}-\varphi \circ y_{n_{i}}\right\| d \mu \leqq a / 3$, and

(c) $\int_{S_{i}}\left\|\varphi \circ x_{n_{i}}-\varphi \circ y_{n_{i}}\right\| d \mu \geqq 2 a / 3$.

Let now $\delta_{k}$ be a positive number such that for $E \in \Sigma$ if $\mu(E) \leqq 2 \delta_{k}$ then $\int_{E}\left\|\varphi \circ x_{n_{k}}-\varphi \circ y_{n_{k}}\right\| d \mu \leqq a / 3$. Let $n_{k+1}>n_{k}$ be so chosen that there exists a measurable set $T_{0}, \mu\left(X \sim T_{0}\right) \leqq \delta_{k}$, and for $\xi \in T_{0},\left\|\varphi(t, \xi)-\varphi\left(t^{\prime}, \xi\right)\right\| \leqq a / 3 \mu(X)$ if $\left|t-t^{\prime}\right|$ $\leqq 1 / n_{k+1}$ and $t, t^{\prime} \in[-d, d]$. Let $X \sim T_{0}=S_{k+1}$. It is verified that

$$
\begin{gathered}
\int_{S_{k+1}}\left\|\varphi \circ x_{n_{k+1}}-\varphi \circ y_{n_{k+1}}\right\| d \mu \geqq 2 a / 3, \\
\int_{S_{k+1}}\left\|\varphi \circ x_{n_{k}}-\varphi \circ y_{n_{k}}\right\| d \mu \leqq a / 3
\end{gathered}
$$

and

$$
\mu\left(S_{k+1}\right) \leqq \delta_{k} \leqq \frac{1}{2} \mu\left(S_{k}\right)
$$


Thus there exist sequences of functions $\left\{x_{n_{l}}\right\},\left\{y_{n_{t}}\right\}$, of corresponding measurable sets $\left\{S_{i}\right\}$, and of positive numbers $\delta_{i}$ such that the conditions listed as (a), (b) and (c) above hold for all $i \geqq 1$. Further it is verified that

$$
\mu\left(\bigcup_{l>k+1} S_{l}\right) \leqq 2 \mu\left(S_{k+1}\right) \leqq 2 \delta_{k} .
$$

Hence if $R_{k}=S_{k} \sim \bigcup_{l=k+1} S_{l}$ then $\left\{R_{k}\right\}_{k>1}$ is a disjoint sequence of measurable sets and from (b) it follows that

$$
\int_{R_{k}}\left\|\varphi \circ x_{n_{k}}-\varphi \circ y_{n_{k}}\right\| d \mu \geqq \frac{a}{3} .
$$

Let $x=\sum_{k \geqq 1} x_{n_{k}} I_{R_{k}}$ and $y=\sum_{k \geqq 1} y_{n_{k}} I_{R_{k}}$. Since $\varphi(x)-\varphi(y)$ is in $L_{B}^{1},\|\varphi(x)-\varphi(y)\|$ is $\mu$-summable. However, the inequality $\left({ }^{*}\right)$ clearly contradicts the summability of the function $\|\varphi(x)-\varphi(y)\|$. Thus the theorem holds for $\mu(x)<\infty$.

Let more generally $\mu(X)=\infty$ and assume that there exist sequences as described in (A). We proceed to construct subsequences $\left\{x_{n_{k}}\right\},\left\{y_{n_{k}}\right\}$ and a sequence of disjoint sets $\left\{R_{k}\right\}$ of finite measure such that $\int_{R_{k}}\left\|\varphi \circ x_{n_{k}}-\varphi \circ y_{n_{k}}\right\| d \mu>a / 2$. Let $R_{1}$ be a set of finite measure such that $\int_{R_{1}}\left\|\varphi \circ x_{1}-\varphi \circ y_{1}\right\| d \mu>a / 2$. The existence of $R_{1}$ is assured by (A). Then the result for the case $\mu(X)<\infty$ implies that, for $n_{2}$ sufficiently large, $\int_{R_{1}}\left\|\varphi \circ x_{n_{2}}-\varphi \circ y_{n_{2}}\right\| d \mu<a / 2$. Hence there exists a set $R_{2} \subset X \sim R_{1}$, $0 \leqq \mu\left(R_{2}\right)<\infty$, such that $\int_{R_{2}}\left\|\varphi \circ x_{n_{2}}-\varphi \circ y_{n_{2}}\right\| d \mu>a / 2$. Again since $\mu\left(R_{1} \cup R_{2}\right)<\infty$ our earlier result implies that for $n_{3}$ sufficiently large $\int_{R_{1} \cup R_{2}}\left\|\varphi \circ x_{n_{3}}-\varphi \circ y_{n_{3}}\right\| d \mu$ $<a / 2$. Hence there exists a set $R_{3} \subset X \sim\left(R_{1} \cup R_{2}\right)$ such that $\mu\left(R_{3}\right)<\infty$ and $\int_{R_{3}}\left\|\varphi \circ x_{n_{3}}-\varphi \circ y_{n_{3}}\right\| d \mu>a / 2$. Proceeding we arrive at sequences of functions $\left\{x_{n_{k}}\right\},\left\{y_{n_{k}}\right\}$ and disjoint sets $\left\{R_{k}\right\}_{k \geqq 1}$ as claimed. It is clear that if $x=\sum_{k \geqq 1} x_{n_{k}} I_{R_{k}}$ and $y=\sum_{k \geqq 1} y_{n_{k}} I_{R_{k}}$ then $\|\varphi \circ x-\varphi \circ y\|$ is not $\mu$-summable, contradicting the fact that $x \rightarrow \varphi \circ x$ maps $L^{\infty}(\mu) \rightarrow L_{B}^{1}$. This completes the proof of Theorem 2 .

The representation result established in the preceding theorems can be carried over to the Banach spaces $L^{p}(\mu), 1 \leqq p<\infty$, of equivalence classes of $p$-summable real valued measurable functions, and this is done in Theorem 3. The proof is similar in details to that of Theorem 2 in [6] and hence the details are not supplied. Note that since $p<\infty, F_{t}$ is now defined only for sets of finite measure.

TheOREM 3. Let $1 \leqq p<\infty$. Suppose that $(X, \Sigma, \mu)$ is a $\sigma$-finite measure space and $F: L^{p}(\mu) \rightarrow B$ is an additive function satisfying

(1) $F$ is continuous on $L^{p}(\mu)$,

(2) for each real number $t, F_{t}$ has almost locally compact average range,

(3) for each real number $t$ and each $T \in \Sigma$ satisfying $\mu(T)<\infty, V\left(F_{t}\right)(T)<\infty$,

(4) for each $T \in \Sigma$ satisfying $\mu(T)<\infty,\left\{F_{t}\right\}_{t \in R}$ when restricted to subsets of $T$ is locally uniformly continuous in variation.

Then there exists a function $\varphi \in \operatorname{Car} B$ such that the mapping $x \rightarrow \varphi \circ x$ takes $L^{p}(\mu)$ into $L_{B}^{1}$, and for $x \in L^{p}(\mu)$

$$
F(x)=\int_{x} \varphi \circ x d \mu .
$$


Moreover $\varphi$ can be taken to satisfy (a) $\varphi(0, \cdot)=0$ a.e. and is then unique up to sets of the form $R \times N$ with $N$ a null set in $X$.

Conversely for every $\varphi \in \operatorname{Car} B$ such that (a) holds and the mapping $x \rightarrow \varphi \circ x$ takes $L^{p}(\mu) \rightarrow L_{B}^{1}$, the formula $\left(^{*}\right)$ defines a $B$-valued additive function $F$ satisfying the conditions (1), (2), (3) and (4).

2. $C$-differentiable additive set functions. Motivated in part by the classical Lebesgue theorem on the derivatives of additive set functions, several authors have studied the differentials of vector valued additive set functions. See for example Clarkson [1], Pettis [8] and Phillips [9]. For convenience of reference we state here a theorem in [9] after recalling pertinent definitions.

Let $G$ be an additive function defined on the class $\Sigma$ of Lebesgue measurable sets in $(0,1)$ into a Banach space $B$. If $\xi \in(0,1)$ then a vector $f(\xi) \in B$ is said to be the derivative of $G$ at $\xi$ if $\lim _{\mu(J) \rightarrow 0}\|G(J) / \mu(J)-f(\xi)\|=0$ where $J$ is an arbitrary interval containing $\xi$ and $\mu$ is Lebesgue measure. If the derivative exists at almost all points $\xi \in(0,1)$ then $G$ is said to be (Lebesgue) differentiable and the function $f$ which is defined a.e. is called the derivative (pseudo-derivative according to [9]) of $G$. If $G: R \times \Sigma \rightarrow B$ is a function such that $G(t, \cdot)$ is a differentiable additive function with $f_{t}$ as the derivative then $G$ is said to be Carathéodory differentiable ( $C$-differentiable) if there exists a null set $N$ such that for all $\xi \notin N$ the mapping $t \rightarrow f_{t}(\xi)$ is a continuous function on $R \rightarrow B$. We proceed to state Theorem 8.1 of [9], which characterizes differentiable set functions of finite variation, in a partial form suitable for our purpose.

THEOREM [Phillips]. A sufficient condition that an additive function $G$ on measurable sets in $(0,1)$ to a Banach space $B$ be differentiable and of finite variation is that $G$ be a Bochner integral. $G$ is then the Bochner integral of its derivative.

We proceed to state the next theorem after establishing a lemma. Throughout this section $\mu$ is Lebesgue measure on $(0,1)$.

LEMMA 4. Let $F$ be an additive function on $L^{\infty}(\mu)$ into a Banach space B. Suppose $x_{n} \rightarrow x$ boundedly a.e. implies $\left\|F\left(x_{n}\right)-F(x)\right\| \rightarrow 0$. Then the following statements are equivalent:

(a) The function $F$ satisfies conditions (1), (2), (3) and (4) in Theorem 1.

(b) If $G: R \times \Sigma \rightarrow B$ is the function $G(t, E)=F\left(I_{E}\right)$ then $G$ is $C$-differentiable.

Proof. If $F$ has property (a) then by Theorem 1 there exists a function $\varphi \in \operatorname{Car} B$ such that $F\left(t I_{E}\right)=\int_{E} \varphi(t, \xi) d \mu(\xi)$. Hence by Phillips theorem, for each real number $t, G(t, \cdot)$ is differentiable at $\xi, \mu$ a.e. Since $\varphi \in$ Car $B$ it follows that (a) $\Rightarrow$ (b). Next let us assume $F$ satisfies the condition in (b). It follows that there exists a function $\varphi \in \operatorname{Car} B$ such that for all simple functions $x$

$$
F(x)=\int_{(0,1)} \varphi(x(\xi), \xi) d \mu(\xi)
$$


Since $F$ is continuous relative to bounded convergence a.e., proceeding as in the proof of Theorem 2 the equation $\left(^{*}\right)$ is verified for all $x \in L^{\infty}(\mu)$. But then it follows from Theorem 2 that $F$ has all the properties stated in (a).

From the preceding lemma we deduce the following representation theorem.

THEOREM 4. Let $F$ be an additive function on $L^{\infty}(\mu) \rightarrow B$ satisfying the condition (1) in Theorem 1. Then $F$ admits an integral representation with a kernel $\varphi \in \operatorname{Car} B$ if and only if the function $G$ in (b) of Lemma 4 is C-differentiable.

3. An example. In this section we discuss an example to show that the representation Theorems 1 and 3 are false if we drop the hypothesis (4) on the additive function $F$. This also shows that in the preceding representation theorem we need the $C$-differentiability of the function $G$.

EXAMPLE. There exists a continuous additive function $F: L^{\infty}(\mu) \rightarrow H$, where the measure space is the unit interval with Lebesgue measure and $H$ is a separable Hilbert space, such that $F$ satisfies the conditions (1), (2) and (3) of Theorem 1 and is uniformly continuous on bounded subsets of $L^{\infty}(\mu)$. However, $F$ does not admit an integral representation with a kernel $\varphi \in \operatorname{Car} H$.

Let $I_{n}$ denote the rectangle $\left(1 / 2^{n}, 1 / 2^{n-1}\right] \times(0,1]$ in the cartesian plane for $n \geqq 1$. Let $\left\{J_{n m}\right\}_{1 \leqq m \leqq 2^{n}}$ be a subdivision of $I_{n}$ into $2^{n}$ rectangles where

$$
J_{n m}=\left(\frac{1}{2^{n}}, \frac{1}{2^{n-1}}\right] \times\left(\frac{m-1}{2^{n}}, \frac{m}{2^{n}}\right], \quad 1 \leqq m \leqq 2^{n} .
$$

Next for each pair $(n, m), n \geqq 1,1 \leqq m \leqq 2^{n}$, let $\mathcal{O}_{n m}$ be a continuous real valued function on $R \times(0,1]$ satisfying the three conditions listed in (I) below:

(a) support $\mathcal{O}_{n m} \subset$ interior $J_{n m}$,

(b) $\int \mathcal{O}_{n m}\left(\frac{2^{-n}+2^{1-n}}{2}, \xi\right) d \mu(\xi)=\frac{1}{2^{n}}$,

(c) $0 \leqq \mathcal{O}_{n m}(t, \xi) \leqq 2$.

Let $\left\{e_{n m}\right\}, n \geqq 1,1 \leqq m \leqq 2^{n}$, be an orthonormal system in the Hilbert space $H$. Define $\varphi: R \times(0,1] \rightarrow H$ as follows:

$$
\varphi=\sum_{n, m} e_{n m} \mathcal{O}_{n m} .
$$

Thus $\varphi$ is continuous except at those points $(h, \xi)$ with $h=0$, and is identically zero on $\{(-\infty, 0] \cup[1, \infty)\} \times(0,1]$.

We proceed to show that the function $F: L^{\infty}(\mu) \rightarrow H$ given by

$$
F(x)=\int_{[0,1]} \varphi \circ x(\xi) d \mu(\xi)
$$

where $\varphi \circ x(\xi)=\varphi(x(\xi), \xi)$ satisfies conditions (1), (2), (3) of Theorem 1 but not (4).

Since it is readily verified that $F$ is additive we proceed to show $F$ satisfies condition (1). We note first that the function $\varphi \circ x$ is Bochner measurable whenever $x$ 
is a measurable real valued function. This follows by the same argument used for $\varphi$ 's in Car $H$, once one restricts attention to the support of $x$. Moreover since, by (I)(c), $\|\varphi \circ x(\xi)\| \leqq 2$ it follows that $\varphi \circ x$ is Bochner integrable. Now for each $\varepsilon>0$ there exists an integer $N$ such that

$$
\left\|\int_{[0,1]} \varphi \circ x d \mu\right\|<\varepsilon / 3 \quad \text { whenever }\|x\|_{\infty} \leqq 1 / 2^{N} .
$$

For according to (II)

$$
\int \varphi \circ x d \mu=\sum_{n \geqq N+1 ; 1 \geqq m \geqq 2^{n}} C_{n m} e_{n m}, \quad C_{n m} \geqq 0,
$$

where

$$
C_{n m}=\int_{[0,1]} \mathcal{O}_{n m}(x(\xi), \xi) d \mu(\xi) \leqq 2 \mu\left(E_{n m}\right)
$$

and

$$
\begin{aligned}
E_{n m} & =\left\{\xi \mid(x(\xi), \xi) \in J_{n m}\right\} \\
& =\left\{\xi \mid x(\xi) \in\left(1 / 2^{n}, 1 / 2^{n+1}\right]\right\} \cap\left((m-1) / 2^{n}, m / 2^{n}\right] .
\end{aligned}
$$

Since the $E_{n m}$ are disjoint and $\bigcup E_{n m} \subset(0,1]$ it follows that

$$
\sum_{n \geqq N+1 ; 1 \leqq m \leqq 2^{n}} C_{n m} \leqq 2 \mu((0,1])=2 .
$$

Moreover by (I)(c)

$$
C_{n m} \leqq 2 \mu\left(\left((m-1) / 2^{n}, m / 2^{n}\right]\right) \leqq 2^{-N},
$$

since $n \geqq N+1$. Thus

$$
\left\|\int_{[0,1]} \varphi \circ x d \mu\right\|^{2}=\sum_{n \geqq N+1 ; 1 \leqq m \leqq 2^{n}} C_{n m}^{2} \leqq 2^{-N} \sum C_{n m} \leqq 2^{1-N},
$$

which in turn is less than $\varepsilon / 3$ for sufficiently large $N$.

Suppose now that the sequence $\left\{x_{n}\right\}$ in $L^{\infty}(\mu)$ converges boundedly a.e. to $x \in L^{\infty}(\mu)$. Since $\varphi(h, \xi)=0$ for $h<0$, it follows that $F(x)=F\left(x^{+}\right), F\left(x_{n}\right)=F\left(x_{n}^{+}\right)$, and hence we may assume that $x$ and $\left\{x_{n}\right\}$ are nonnegative. With $N$ as in the preceding paragraph, select a real number $h_{0}, 2^{-N-1}<h_{0}<2^{-N}$, such that $x^{-1}\left(\left\{h_{0}\right\}\right)$ is a null set. Then with $x^{\prime}, x^{\prime \prime}, x_{n}^{\prime}, x_{n}^{\prime \prime}$ defined by

$$
\begin{array}{ll}
x^{\prime}=x I_{x^{-1}\left(\left(h_{0}, \infty\right)\right),} & x^{\prime \prime}=x I_{x^{-1}\left(\left[0, h_{0}\right)\right)} \\
x_{n}^{\prime}=x_{n} I_{x_{n}^{-1}\left(\left(h_{0}, \infty\right)\right),} & x_{n}^{\prime \prime}=x_{n} I_{x_{n}^{-1}\left(\left[0, h_{0}\right)\right),}
\end{array}
$$

the following relations hold: $x_{n}^{\prime} \rightarrow x^{\prime}$ boundedly a.e., $\left\|x_{n}^{\prime \prime}\right\|_{\infty},\left\|x^{\prime \prime}\right\|_{\infty} \leqq 2^{-N}$. Thus

$$
\begin{aligned}
\left\|F\left(x_{n}\right)-F(x)\right\| & \leqq\left\|F\left(x_{n}^{\prime}\right)-F\left(x^{\prime}\right)\right\|+\left\|F\left(x_{n}^{\prime \prime}\right)\right\|+\left\|F\left(x^{\prime \prime}\right)\right\| \\
& \leqq 2 \varepsilon / 3+\left\|F\left(x_{n}^{\prime}\right)-F\left(x^{\prime}\right)\right\|,
\end{aligned}
$$


by definition of $N$. Noting that $\varphi$ restricted to $\left[2^{-N-1}, \infty\right) \times[0,1]$ coincides there with a Carathéodory function, we deduce from Theorem 2 that $\left\|F\left(x_{n}^{\prime}\right)-F\left(x^{\prime}\right)\right\| \rightarrow 0$. Thus for sufficiently large $n,\left\|F\left(x_{n}\right)-F(x)\right\|<\varepsilon$, which shows that condition (1) holds.

Since for each real number $t$ the set function $F_{t}$ is a Bochner integral, it follows by the main theorem in [10] that conditions (2) and (3) hold.

However, $F$ fails to satisfy condition (4). For from (I)(b) it follows that if $h_{n}=\frac{1}{2}\left(2^{-n}+2^{1-n}\right)$ then $\int\left\|\varphi\left(h_{n}, \xi\right)-\varphi(0, \xi)\right\| d \mu(\xi)=\int\left\|\varphi\left(h_{n}, \xi\right)\right\| d \mu(\xi)=2^{n} \cdot 1 / 2^{n}=1$, although $h_{n} \rightarrow 0$.

Let now $M$ be a positive real number. We wish to show that $F$ is uniformly continuous on the ball $S_{M}=\left\{x \mid x \in L^{\infty}(\mu),\|x\|_{\infty} \leqq M\right\}$. If $F$ is not uniformly continuous on $S_{M}$ then there exist two sequences $\left\{x_{n}\right\},\left\{y_{n}\right\}$ with $\left\|x_{n}-y_{n}\right\|_{\infty} \rightarrow 0$ and $\left\|F\left(x_{n}\right)-F\left(y_{n}\right)\right\|>\varepsilon$ for some positive number $\varepsilon$ independent of $n$. As above, we can assume that $x_{n}, y_{n}$ are nonnegative. Select $N$ as in the preceding paragraphs. We decompose each of the functions $x_{n}, n \geqq 1$, as $x_{n}=x_{n}^{\prime}+x_{n}^{\prime \prime}$ with $x_{n}^{\prime}, x_{n}^{\prime \prime}$ having disjoint supports $A_{n}^{\prime}$ and $A_{n}^{\prime \prime}$. Here $\left\|x_{n}^{\prime \prime}\right\|_{\infty} \leqq \frac{1}{2} 2^{-N}$ and $x_{n}^{\prime}(\xi)>\frac{1}{2} 2^{-N}$ for all $\xi$ in its support $A_{n}^{\prime}$. Define $y_{n}^{\prime}=y_{n} I_{A_{n}^{\prime}}, y_{n}^{\prime \prime}=y_{n}-y_{n}^{\prime}$. Then for $n$ sufficiently large $0 \leqq y_{n}^{\prime \prime}<2^{-N}$ and $y_{n}^{\prime} \geqq \frac{1}{4} 2^{-N}$ on $A_{n}^{\prime}$. Now consider

$$
\begin{aligned}
\left\|F\left(x_{n}\right)-F\left(y_{n}\right)\right\| & \leqq\left\|F\left(x_{n}^{\prime}\right)-F\left(y_{n}^{\prime}\right)\right\|+\left\|F\left(x_{n}^{\prime \prime}\right)\right\|+\left\|F\left(y_{n}^{\prime \prime}\right)\right\| \\
& \leqq 2 \varepsilon / 3+\left\|F\left(x_{n}^{\prime}\right)-F\left(y_{n}^{\prime}\right)\right\|
\end{aligned}
$$

by definition of $N$. Let us examine the last term. Now $x_{n}^{\prime}, y_{n}^{\prime} \geqq \delta=\frac{1}{4} 2^{-N}$ on $A_{n}^{\prime}$. Noting that $\varphi$ restricted to $[\delta, \infty) \times(0,1]$ coincides there with a Carathéodory function we deduce from Theorem 2 that $\left\|F\left(x_{n}^{\prime}\right)-F\left(y_{n}^{\prime}\right)\right\| \rightarrow 0$, leading to a contradiction.

Since $F$ is uniformly continuous on the sets $S_{M}$ and yet does not satisfy condition (4), we see that the converse of Lemma 1 fails (see Remark 1(a) in §1).

4. Representation of additive functions on $L_{E}^{p} \rightarrow B$. In the present section we give generalized forms of the preceding representation theorems, namely the theorems which occur on replacing the real line $R$ by an infinite-dimensional separable Banach space $E$.

Let $(X, \Sigma, \mu)$ be a complete $\sigma$-finite positive measure space and $E, B$ be two Banach spaces. As already noted $L_{E}^{p}(1 \leqq p<\infty)$ is the Banach space of equivalence classes of $E$-valued Bochner measurable functions $f$ on $X$ such that the real valued function $x \rightarrow\|f(x)\|^{p}$ is $\mu$-summable. Similarly $L_{E}^{\infty}$ is the Banach space of equivalence classes of $E$-valued Bochner measurable functions $f$ with the norm defined

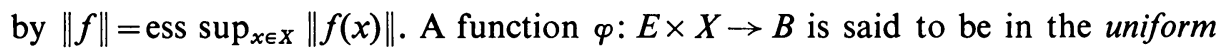
Carathéodory class relative to $E$ on $X \rightarrow B$, in brief $\varphi \in U-\operatorname{Car}(E, B)$, if $\varphi(e, \cdot)$ is a $B$-valued Bochner measurable function for each vector $e \in E$ and $\varphi(\cdot, \xi)$ is uniformly continuous on bounded subsets of $E$ for all $\xi$ outside a $\mu$-null set. Given a $p, 1 \leqq p \leqq \infty, \varphi \in U-\operatorname{Car}(E, B)$ is said to be in $U-\operatorname{Car}^{p}(E, B)$ if the composition operator $x \rightarrow \varphi \circ x$ where $\varphi \circ x(\xi)=\varphi(x(\xi), \xi)$ maps $L_{E}^{p}$ into $L_{B}^{1}$. 
Before proceeding to the main theorem of this section let us note that the following properties of the real line $R$ were used in the proofs of the theorems in $\$ 1$. (1) The separability of the real line was used in the proof of Lemma 3 preceding Theorem 1. (2) The local compactness of the real line was involved in the proof of Theorem 2 in order to conclude that the functions $\varphi(\cdot, \xi): R \rightarrow B$ are uniformly continuous on bounded subsets of the real line. With the aid of these observations it is easily verified that the theorems stated in $§ 1$ remain valid (exactly as formulated) when $R$ is replaced by any finite-dimensional Banach space $E\left({ }^{1}\right)$. On the other hand, for the infinite-dimensional case we have the following result. [In analogy with the terminology in $\S 1$ we let $F_{e}$ denote the set function defined on $\Sigma \rightarrow B$ (or only on sets in $\Sigma$ of finite measure) by the equation $F_{e}(M)=F\left(e I_{M}\right)$.]

Theorem 5. Let $(X, \Sigma, \mu)$ be as in Theorem 1 , let E be a separable Banach space, and let $B$ be an arbitrary Banach space. Let $F: L_{E}^{p} \rightarrow B(1 \leqq p \leqq \infty)$ be an additive function satisfying the following conditions:

1. For each vector $e \in E$ the set function $F_{e}$ which carries sets in $\Sigma$ of finite measure to $B$ has almost locally compact average range.

2. For each $e \in E$, if $T \in \Sigma, \mu(T)<\infty$, then $V\left(F_{e}\right)(T)<\infty$.

3. On each set $T$ of finite measure, the family of set functions $\left\{F_{e}\right\}_{e \in E}$ is locally uniformly continuous in variation.

4. $F$ is continuous relative to $L_{E}^{p}$ norm, if $p<\infty$, and is continuous with respect to bounded a.e. convergence if $p=\infty$.

Then there exists a function $\varphi \in U-\operatorname{Car}^{p}(E, B)$ such that

$$
F(x)=\int_{X} \varphi \circ x d \mu \quad \text { for } x \in L_{E}^{p}
$$

Moreover $\varphi$ can be taken to satisfy

$$
\varphi(0, \cdot)=0 \text { a.e. }
$$

and is then unique up to sets of the form $E \times N$ with $N$ a null set in $X$.

Conversely, for any $\varphi \in U-\operatorname{Car}^{p}(E, B)$ satisfying (a), (*) defines an additive function from $L_{E}^{p} \rightarrow B$ satisfying conditions $1,2,3$ and 4 .

The proof of Theorem 5 is very similar to the proofs of Theorems 1,2 and 3 and is easily supplied with the aid of observations (1) and (2) in the paragraph preceding the statement of the present theorem. Hence we omit the details.

As noted earlier, when $B=R$ the following result for linear functionals appears as a corollary. Here $E^{*}$ denotes the dual of $E$, and a function $f: X \rightarrow E^{*}$ is said to be weakly measurable provided that (a) for some null set $N$ the $w^{*}$-closure of $f(X \sim N)$ is separable in the $w^{*}$-topology, and (b) $f^{-1}(G)$ is measurable for each $w^{*}$-open set $G \subset E^{*}$.

(1) Likewise for the theorems in [6] and [2]. 
Theorem 6 (Dieudonné [13], [14], Edwards [4, TheOrem 8.20.3]). Suppose that $E$ is a separable Banach space and $F$ is a continuous linear functional on $L_{E}^{p}$, where $1 \leqq p<\infty$. Then there exists a function $f$ mapping $X$ into $E^{*}$ that is weakly measurable and such that

$$
\begin{gathered}
F(x)=\int\langle f, x\rangle d \mu \text { for each } x \in L_{E}^{p}, \\
\|F\|=\left[\int\|f\|^{p^{\prime}} d \mu\right]^{1 / p^{\prime}}, \quad \text { where } 1 / p^{\prime}+1 / p=1, \quad p>1, \\
=\text { ess sup }\|f(\cdot)\|, \quad p=1 .
\end{gathered}
$$

Conversely any weakly measurable function $f$ for which the right-hand side of $(\beta)$ is finite defines, via $(\alpha)$, a continuous linear functional $F$ on $L_{E}^{p}$ for which $(\beta)$ holds.

In order to obtain the proof we develop certain preliminary results.

LEMMA 5. If $F$ is a continuous linear functional on $L_{E}^{p}$, where $1 \leqq p<\infty$, then $F$ satisfies conditions 1, 2, 3, 4 of Theorem 5.

Proof. Since $F$ is linear it is verified that $F$ is an additive function on $L_{E}^{p} \rightarrow R$. Thus for each vector $e \in E, F_{e}$ is an additive set function on sets $T \in \Sigma$ such that $\mu(T)<\infty$. Let $\Sigma_{T}=\{T \cap M \mid M \in \Sigma\}$. Since $F$ is continuous and $F(0)=0$ it is verified that $F_{e} \mid \Sigma_{T}$ is $\mu$-absolutely continuous. Hence by the (ordinary) Radon-Nikodym Theorem and the main theorem in Rieffel [10] (for real measures) we infer that $F$ has properties 1 and 2 . Condition 4 holds by hypothesis.

We proceed to verify condition 3 . Let $T \in \Sigma, \mu(T)<\infty$, and $\varepsilon>0$ be given. Let $\delta=\varepsilon /\|F\| \mu(T)^{1 / p}(1+\varepsilon)$. Now suppose $\left\{T_{i}\right\}_{1 \leqq i \leqq n}$ is a measurable partition of $T$ and $\left\{e_{i}\right\}_{1 \leqq i \leqq n},\left\{e_{i}^{\prime}\right\}_{1 \leqq i \leqq n}$ in $E$ satisfy $\left\|e_{i}-e_{i}^{\prime}\right\| \leqq \delta$. Then by the definition of the variation, there exist partitions $\left\{T_{i j}\right\}_{1 \leqq j \leqq m_{i}}$ of the $\left\{T_{i}\right\}$ such that

$$
\begin{aligned}
\sum_{i} V\left(F_{e_{i}}-F_{e_{i}}\right)\left(T_{i}\right) & \leqq(1+\varepsilon) \sum_{i} \sum_{j}\left|F_{e_{i}}\left(T_{i j}\right)-F_{e_{i}^{i}}\left(T_{i j}\right)\right| \\
& =(1+\varepsilon) \sum_{i, j}\left|F_{e_{i}-e_{i}}\left(T_{i j}\right)\right| .
\end{aligned}
$$

Let

$$
\begin{aligned}
& P=\left\{(i, j) \mid 1 \leqq i \leqq n, 1 \leqq j \leqq m_{i} \text { and } F_{e_{i}-e_{i}}\left(T_{i j}\right) \geqq 0\right\} \\
& Q=\left\{(i, j) \mid 1 \leqq i \leqq n, 1 \leqq j \leqq m_{i} \text { and } F_{e_{i}-e_{i}^{i}}\left(T_{i j}\right)<0\right\}
\end{aligned}
$$

Then

$$
\begin{aligned}
\sum_{i, j}\left|F_{e_{i}-e_{i}^{\prime}}\left(T_{i j}\right)\right| & =\sum_{(i, j) \in P} F_{e_{i}-e_{i}}\left(T_{i j}\right)-\sum_{(i, j) \in Q} F_{e_{i}-e_{i}^{\prime}}\left(T_{i j}\right) \\
& =F\left(\sum_{(i, j) \in P}\left(e_{i}-e_{i}^{\prime}\right) I_{T_{i j}}-\sum_{(i, j) \in Q}\left(e_{i}-e_{i}^{\prime}\right) I_{T_{i j}}\right) \\
& \leqq\|F\| \delta\left(\sum \mu\left(T_{i j}\right)\right)^{1 / p} .
\end{aligned}
$$


It follows from (1) and (2) that

$$
\sum V\left(F_{e_{i}-e_{i}}\right)\left(T_{i}\right) \leqq(1+\varepsilon)\|F\|(\mu(T))^{1 / p} \delta<\varepsilon .
$$

Hence $F$ satisfies condition 3 .

LEMMA 6. If for some $\varphi \in U-\operatorname{Car}^{p}(E, R)$ the functional $F$ defined on $L_{E}^{p}$ by

$$
F(x)=\int_{X} \varphi(x(\xi), \xi) d \mu(\xi)
$$

is linear, then there exists $a \mu$-null set $N$ and $a w^{*}$-measurable function $f: X \rightarrow E^{*}$, with $\|f(\cdot)\| \mu$-measurable, such that $\varphi(e, \xi)=f(\xi)(e)$ for $e \in E, \xi \notin N$.

Proof. Since $F$ is linear it is verified that if $A \in \Sigma, \mu(A)<\infty$, then for any two vectors $e, e^{\prime} \in E$,

$$
\begin{aligned}
\int_{A} \varphi\left(e+e^{\prime}, \xi\right) d \mu(\xi) & =F\left(\left(e+e^{\prime}\right) I_{A}\right)=F\left(e I_{A}\right)+F\left(e^{\prime} I_{A}\right) \\
& =\int_{A} \varphi(e, \xi) d \mu(\xi)+\int_{A} \varphi\left(e^{\prime}, \xi\right) d \mu(\xi) .
\end{aligned}
$$

Hence by the uniqueness of Radon-Nikodym derivatives it follows that $\varphi\left(e+e^{\prime}, \xi\right)$ $=\varphi(e, \xi)+\varphi\left(e^{\prime}, \xi\right)$ a.e. Since $E$ is separable and $\varphi \in U-\operatorname{Car}(E, B)$ it is further true that there exists a $\mu$-null set $N_{1}$ such that for all $\xi \notin N_{1}$, and for any pair $e, e^{\prime} \in E$, $\varphi\left(e+e^{\prime}, \xi\right)=\varphi(e, \xi)+\varphi\left(e^{\prime}, \xi\right)$. It follows from this that, for $\xi \notin N_{1}, \varphi(c e, \xi)=c \varphi(e, \xi)$ whenever $c$ is rational. Hence, since the rationals are dense in $R$ and $\varphi \in U$ $-\operatorname{Car}(E, B)$ one has that for all $e \in E$ and all $c \in R, \varphi(c e, \xi)=c \varphi(e, \xi)$ for $\xi$ outside a null set $N \supset N_{1}$. Moreover, without loss of generality we can assume $N$ to be such that $\varphi(\cdot, \xi)$ is uniformly continuous for $\xi \notin N$. Thus if $f(\xi)$ is the function on $E \rightarrow R$ defined by $f(\xi)(e)=\varphi(e, \xi)$ then $f(\xi) \in E^{*}$ for all $\xi \notin N$ and, by redefining $\varphi$ so that $\varphi(E \times N)=0$, we can assume that $f(\xi) \in E^{*}$ for all $\xi \in X$. Further for each $e \in E$, $f(\cdot)(e)=\varphi(e, \cdot)$ is a real valued measurable function on $X$. Thus since $E$ is separable it follows from Proposition 8.15.3 of [4] that $f: X \rightarrow E^{*}$ is a $w^{*}$-measurable function and that the function $\xi \rightarrow\|f(\xi)\|$ is a real valued measurable function.

Proof of the theorem. By Lemma $5, F$ satisfies the hypotheses of Theorem 5. Hence $F$ has a representation of the form (*) with $\varphi \in U-\operatorname{Car}^{p}(E, R)$. By Lemma 6 this yields the formula

$$
F(x)=\int f(\xi)(x(\xi)) d \mu(\xi)
$$

with $f: X \rightarrow E^{*}$ satisfying the measurability conditions stated in the lemma.

We now proceed to verify equation $(\beta)$. Let $U_{n}$ be the closed ball in $E^{*}$ of radius $n(n \geqq 1)$. Let $C_{n}$ be the measurable set $f^{-1}\left(U_{n}\right)$. Then $\bigcup_{n \geqq 1} C_{n}=X$ and $f \mid C_{n}$ is a $w^{*}$-pointwise almost uniform limit of a sequence of countably valued functions 
$\left\{l_{n}^{i}\right\}_{i \geqq 1}$ with support $\left(l_{n}^{i}\right) \subset C_{n}$. This follows from the metrizability of $U_{n}$ in the $w^{*}$-topology since by its measurability $f$ is almost separably valued. Utilizing this fact it is easy to show that there exists for each positive $\varepsilon$ a countably valued function $x^{n}: X \rightarrow E$ with support in $C_{n}$ and unit $L_{E}^{p}$-norm such that

$$
\begin{aligned}
& \|F\| \geqq F\left(x^{n}\right) \geqq\left[\int_{C_{n}}\|f\|^{p^{\prime}}\right]^{1 / p^{\prime}}(1-\varepsilon), \quad p>1, \\
& \geqq \underset{C_{n}}{\operatorname{ess} \sup }\|f(\cdot)\|(1-\varepsilon), \quad p=1 .
\end{aligned}
$$

It follows from this that $\|f\| \in L_{R}^{p^{\prime}}$ and $\|F\|^{p^{\prime}}=\int_{X}\|f\|^{p^{\prime}} d \mu$ (for $p=1,\|F\|$ = ess sup $\|f(\cdot)\|)$.

For the converse note that if $f: X \rightarrow E^{*}$ is a $w^{*}$-measurable function and $\int_{X}\|f\|^{p^{\prime}} d \mu<\infty$ (for $p=1$, ess sup $\|f(\cdot)\|<\infty$ ) then the function $\varphi: E \times X \rightarrow R$ defined by $\varphi(e, \xi)=\langle f(\xi), e\rangle$ is in $U-\operatorname{Car}(E, R)$. The verification that $\varphi \in U$ $-\operatorname{Car}^{p}(E, R)$ and that the linear functional $F: L_{E}^{p} \rightarrow R$ defined by $F(x)$ $=\int\langle f(\xi)(x(\xi))\rangle d \mu(\xi)$ is continuous and satisfies $(\beta)$ follows by the methods of the preceding paragraph.

ACKNOWLEDGEMENTS. We gratefully acknowledge respective partial support of this research by the National Science Foundation under Grant GP 11697 and by a (1969) Scaife Faculty Grant administered by Carnegie-Mellon University. We are grateful to the referee for several useful comments.

\section{REFERENCES}

1. J. A. Clarkson, Uniformly convex spaces, Trans. Amer. Math. Soc. 40 (1936), 396-414.

2. L. Drewnowski and W. Orlicz, On orthogonally additive functionals, Bull. Acad. Polon. Sci. Sér. Sci. Math. Astronom. Phys. 16 (1968), 883-888. MR 39 \#6069.

3. N. Dunford and J. T. Schwartz, Linear operators. I: General theory, Pure and Appl. Math., vol. 7, Interscience, New York, 1958. MR 22 \#8302.

4. R. E. Edwards, Functional analysis. Theory and applications, Holt, Rinehart and Winston, New York, 1965. MR 36 \#4308.

5. M. A. Krasnosel'skiǐ, Topological methods in the theory of non-linear integral equations, GITTL, Moscow, 1956; English transl., Macmillan, New York, 1964. MR 20 \#3464; MR 28 \#2414.

6. V. J. Mizel, Characterization of nonlinear transformations possessing kernels, Canad. J. Math. 22 (1970), 449-471.

7. V. J. Mizel and K. Sundaresan, Representation of additive and biadditive functionals, Arch. Rational Mech. Anal. 30 (1968), 102-126. MR 37 \#3341.

8. B. J. Pettis, Differentiation in Banach spaces, Duke Math. J. 5 (1939), 254-269.

9. R. S. Phillips, Integration in a convex linear topological space, Trans. Amer. Math. Soc. 47 (1940), 114-145. MR 2, 103.

10. M. A. Rieffel, The Radon-Nikodym theorem for the Bochner integral, Trans. Amer. Math. Soc. 131 (1968), 466-487. MR 36 \#5297.

11. W. A. Woyczyński, Additive functionals on Orlicz spaces, Colloq. Math. 19 (1968), 319-326. MR 37 \#5688.

12. V. J. Mizel and K. Sundaresan, Characterization of nonlinear vector valued functions, Report 69-30, Department of Mathematics, Carnegie-Mellon University, Pittsburgh, Pa., 1969. 
13. J. Dieudonné, Sur le théorème de Lebesgue-Nikodym. III, Ann. Univ. Grenoble Sect. Sci. Math. Phys. 23 (1948), 25-53. MR 10, 519.

14. —— Sur le théorème de Lebesgue-Nikodym. V, Canad. J. Math. 3 (1951), 129-139. MR 13, 448.

15. J. Neveu, Bases mathématiques du calcul des probabilités, Masson, Paris, 1964; English transl., Holden-Day, San Francisco, Calif., 1965. MR 33 \#6659; 6660.

\section{Carnegie-Mellon University,}

Pittsburgh, Pennsylvania 15213 